INVESTIGACIÓN/RESEARCH

\title{
LA ESPECIALIZACIÓN PERIODÍSTICA EN PATRIMONIO CULTURAL EN LAS SECCIONES DE LA PRENSA REGIONAL DE CASTILLA Y LEÓN
}

María Monjas Eleta1: Universidad de Valladolid. España. mariamon@hmca.uva.es

\section{RESUMEN}

La información sobre Patrimonio Cultural, por su vinculación con el concepto más amplio de cultura, puede constituir un bloque de especialización dentro de la sección de "Cultura" de la prensa. Sin embargo, su tratamiento periodístico se debate entre su adscripción a la sección de Cultura, lo que implica un mayor nivel de especialización de los redactores, y su inclusión en otras secciones, como las de Sociedad o la de información regional. Este artículo se plantea el estudio de la especialización periodística en Patrimonio Cultural por su ubicación en las secciones de "Castilla y León" y "Cultura" en los dos principales diarios de esta comunidad autónoma: El Norte de Castilla y El Mundo de Castilla y León con el objetivo de averiguar los criterios determinan el reparto de las informaciones en una $u$ otra sección $y$, en definitiva, si existe o no, aunque sea de forma incipiente una especialización del periodismo cultural centrada en los bienes patrimoniales.

PALABRAS CLAVE: Patrimonio cultural - Periodismo especializado - Prensa Cultura - Tratamiento de la información.

\footnotetext{
1 Autor correspondiente:

María Monjas Eleta: Profesora de la Universidad de Valladolid. Miembro del Grupo de Investigación en Nuevas Tendencias de la Comunicación de la Universidad de Valladolid y del Proyecto de Innovación Docente “TIC y multidisciplinareidad en el Grado en Periodismo: medios culturales especializados". España.

Correo: mariamon@hmca.uva.es
} 


\title{
SPECIALIZED JOURNALISM ON CULTURAL HERITAGE. THE SECTIONS OF CASTILLA Y LEON REGIONAL PRESS
}

\begin{abstract}
Information on Cultural Heritage, for its links with the wider concept of culture, can be a block of specialization within the section "Culture" of the press. Nevertheless, his journalistic treatment debates between his adscription to the section of Culture, which implies a major level of specialization of the editors, and his incorporation in other sections, as those of Life or that of regional information. This article presents the study of journalistic specialization in Cultural Heritage for its location in the sections of "Castilla y Leon" and "Culture" in the two principal newspapers in this region: El Norte de Castilla and El Mundo de Castilla y León with the aim to verify the criteria determine the distribution of information in one section or another and, definitively, if it exists or not, though it is of incipient form a specialization of the cultural journalism centred on the patrimonial goods.
\end{abstract}

KEY WORDS: Cultural heritage - Specialized journalism - Press - Culture Information processing.

\section{INTRODUCCIÓN}

El Patrimonio Cultural es una expresión acuñada a partir de la segunda mitad del siglo XX a través de diversos convenios internacionales de protección del patrimonio, principalmente de la Organización de las Naciones Unidas para la Educación, la Ciencia y la Cultura, Unesco, que elaboró una primera definición del concepto en la Convención sobre la protección del patrimonio mundial, cultural y natural de 1972 celebrada en París que progresivamente ha sustituido a la anterior y más restrictiva fórmula de patrimonio histórico-artístico (Fernández de Paz, 2006 y Hernández, 2002).

En la actualidad el Patrimonio Cultural engloba el patrimonio histórico artístico, los bienes muebles e inmuebles que consideramos dignos de conservar para generaciones futuras porque alcanzan un alto grado de excelencia dentro de las artes: pintura, escultura, artes decorativas, arquitectura, entre otras. Además incluye otros objetos no artísticos que conservan las costumbres y formas de hacer, utensilios de distintos oficios o distintas épocas que conforman el patrimonio etnológico, y el patrimonio inmaterial, que puede sustanciarse o no en un objeto y que refleja los valores y creencias de una sociedad.

El concepto de Patrimonio Cultural está abierto y en continua transformación, porque es la sociedad, también en continua evolución, quien otorga valor a un conjunto de bienes. Valor no en un sentido económico, sino un valor simbólico, de representación de los rasgos que conforman la identidad colectiva, como define el prólogo de la Ley de Patrimonio Histórico Español, "porque los bienes que lo integran se han convertido en patrimoniales debido exclusivamente a la acción social que cumplen, directamente derivada del aprecio con que los mismos ciudadanos los han ido revalorizando". En 
este proceso de revalorización de los bienes culturales juegan un papel decisivo los medios de comunicación como agentes de divulgación cultural conformadores de una opinión pública que ponga en valor los elementos de su legado cultural.

La información sobre Patrimonio Cultural abarca, por tanto, muy diversos aspectos y enfoques, según el aspecto que se destaque. Así por ejemplo puede hacerse hincapié en las repercusiones del Patrimonio Cultural en un sector económico fundamental como el turismo o en la inversión pública para la restauración de un bien cultural. De la relación entre patrimonio y política surgen también informaciones que ponen de relieve la confrontación entre partidos tomando como base asuntos del patrimonio o cuestiones relativas a legislación, inversión pública política cultural o subvenciones. La política determina también la fuente principal de la información ya que son las administraciones públicas quienes tienen entre sus obligaciones las de conservar y acrecentar el Patrimonio Cultural.

Otros textos informan sobre exposiciones, restauración de bienes culturales, conmemoraciones históricas, descubrimientos o hallazgos, incluso puede darse un enfoque más relacionado con el ocio dentro del denominado turismo cultural. También, si tenemos en cuenta la constante evolución del concepto adecuándose a la sensibilidad de la sociedad en cada momento encontramos informaciones sobre la solicitud o declaración de un nuevo bien cultural de forma que el catálogo de bienes pertenecientes al Patrimonio Cultural va incrementándose a medida que la sociedad toma conciencia del valor y relevancia en determinados bienes.

La diversidad de asuntos y enfoques de las informaciones sobre Patrimonio Cultural hace necesario un estudio del tratamiento periodístico que se da a estas informaciones que permita comprender la forma en que los medios, concretamente la prensa regional en el caso de la presente investigación, abordan este tema y el grado de especialización necesario para una información sobre el Patrimonio Cultural de calidad que contribuya a la creación de una sociedad "atenta a los avances del conocimiento y responsable con la defensa del patrimonio cultural y natural de todo el planeta" (Casals, 2005: 117).

La necesidad de una mayor especialización es especialmente vital en el caso del Patrimonio Cultural porque la difusión a través de los medios de comunicación puede contribuir no sólo a la concienciación para su conservación sino también, y en primer lugar, para su creación ya que "la 'patrimonialidad' no proviene de los objetos, sino de los sujetos, y el proceso de patrimonialización es un proceso de construcción de la memoria colectiva" (Tugores y Planas, 2006:19) en la que juegan un papel esencial los medios de comunicación por lo que, como señala $\mathrm{M}^{\mathrm{a}}$ Teresa Mercado Sáez (2006), "debe trabajarse en la especialización de estos periodistas capaces de dar una interpretación y una opinión certera sobre cualquier asunto relacionado con el patrimonio cultural" (2006: 233).

Esta investigación se enmarca dentro de una corriente que ha producido en los últimos años un incremento de los estudios que abordan la relación entre los medios de comunicación y el Patrimonio Cultural en su papel de difusores del patrimonio, como 
Patrimonio Cultural - Periodismo especializado - Prensa - Cultura - Tratamiento de la información

en número de 2007 de la revista PH Cuadernos 21 editada por el Instituto Andaluz de Patrimonio Histórico o la obra coordinada por Santos M. Mateos Rusillo (2008). También aparecen referencias de algunas páginas a los medios de comunicación en los manuales sobre patrimonio cultural (Tugores y Planas, 2006 y Hernández, 2002).

En general, estos estudios sobre Patrimonio Cultural y periodismo no siguen una metodología de análisis propia de las Ciencias Sociales. Los escasos trabajos que se basan en análisis de contenido, utilizan para su análisis clasificaciones de las informaciones con tipologías ajenas a los estudios de periodismo por estar realizados por especialistas en otros campos como la Arqueología, (Ruiz Zapatero y Mansilla Castaño, 1999, Rodríguez Temiño, 2007).

El estudio de la bibliografía del ámbito de la Comunicación y Ciencias de la Información referida al Patrimonio Cultural pone de manifiesto la carencia de análisis que permitan entender cuál es el tratamiento periodístico del Patrimonio Cultural y si ese tratamiento se traduce en un área de especialización periodística, como pretende esta investigación. El campo de análisis de este trabajo es el periodismo especializado, concretamente el periodismo cultural en los que encontramos contadas referencias al Patrimonio Cultural como un bloque de especialización periodística (veáse, por ejemplo, Llano, 2008). De la exploración bibliográfica realizada se extraen contados ejemplos de estudio del tratamiento del Patrimonio Cultural en los medios de comunicación con propuestas principalmente teóricas (Mercado Sáez, 2006 y Leyton Rivas, 2007) o centradas en un tipo de patrimonio concreto (Meneses Fernández, 2011).

Este estudio se centra en un ámbito territorial concreto, la Comunidad Autónoma de Castilla y León, debido a que la idea de identidad colectiva está en la base del concepto de Patrimonio Cultural y esa identidad está indisolublemente ligada al territorio, una cuestión de especial relevancia en el contexto de un mundo globalizado y particularmente en España por la descentralización del Estado en las Comunidades Autónomas, donde el Patrimonio Cultural se convierte en una referencia identitaria y diferenciadora (Fernández de Paz, 2006). Castilla y León señala en el artículo 4 de su Estatuto de que "la lengua castellana y el patrimonio histórico, artístico y natural son valores esenciales para la identidad de la Comunidad de Castilla y León" 2 .

\subsection{Patrimonio Cultural, un bloque de especialización periodística.}

La información sobre Patrimonio Cultural, como hemos señalado, se enmarca genéricamente dentro del área o sección de Cultura y dentro de ella constituye un bloque o subcampo de especialización del periodismo cultural por la afinidad en cuanto al lenguaje y las fuentes de las informaciones sobre bienes culturales, según la terminología desarrollada por Carmen Herrero que diferencia entre sección, área y bloque (Herrero Aguado, 2002).

\footnotetext{
${ }^{2}$ Estatuto de Autonomía de Castilla y León, Ley Orgánica 4/1983. El artículo 4 quedó modificado por la Ley Orgánica 4/1999. El artículo 4 mantiene este mismo punto en la reforma del Estatuto de Castilla y León, Ley Orgánica 14/2007 de 30 de noviembre.
} 
El área hace referencia a un ámbito de trabajo, es variable y puede coincidir con el nombre de una sección. La sección también tiene una dimensión de organización interna en la redacción pero es, fundamentalmente y tomando como modelo la prensa escrita, un conjunto de textos e imágenes agrupados bajo un denominador común y que cumple funciones de presentación y ordenación de la lectura. Por último están los bloques que son grupos de texto dentro de las secciones con una afinidad mucho mayor entre sí. Tienen su propio lenguaje, fuentes y estrategias diferenciadas y son el campo más adecuado para la especialización.

La heterogeneidad de manifestaciones que se incluyen dentro del periodismo cultural da lugar a variaciones en cuanto a la ubicación de las informaciones culturales en la estructura de los medios ya que no existe consenso respecto a las informaciones asignadas a ella en aquellos en los que existe un área o sección específica de Cultura.

La distribución de contenidos en las secciones, generalmente, se hace a partir de dos criterios básicos, el origen geográfico (local, regional, nacional e internacional) y el ámbito temático (información política, económica, deportiva, cultural o social). Sin embargo hay ciertos matices que hay que tener en cuenta como por ejemplo que habitualmente se entiende por información internacional o nacional la información política de esas áreas geográficas. Por el contrario, cuando se habla de información local o regional, puede incluir información de cualquier temática del ámbito geográfico más cercano al de la publicación.

Los matices en cuanto a la ordenación de los contenidos en las secciones también abarcan otros aspectos, en el caso concreto de la sección de Cultura. Generalmente, así lo señala Rodríguez Pastoriza (2006), las realizaciones de la alta cultura (bellas artes, literatura, música clásica, etc.) se incluyen en la sección o área de "Cultura" de los medios de comunicación mientras que las manifestaciones de la cultura popular como el folclore o la artesanía junto con otras como moda o gastronomía suelen pertenecer a áreas distintas, principalmente el área de Sociedad que en algunos medios se convierte en una macroárea que absorbe Cultura y Espectáculos. El enfoque dado a una información cultural también puede suponer que una misma información pueda ubicarse en diferentes secciones dependiendo de su enfoque.

Por todo lo señalado anteriormente, la información sobre el Patrimonio Cultural se enmarca dentro del área o sección de Cultura y dentro de ella podría constituir un bloque o subcampo de especialización del periodismo cultural por la afinidad en el lenguaje y las fuentes de las informaciones sobre bienes culturales. Así, el Patrimonio Cultural sería un bloque especializado de la sección de Cultura como puede serlo sucesos o educación dentro de la sección de Sociedad o fútbol, tenis, ciclismo... dentro de la de Deportes, incluso Parlamento o Partidos dentro de la de Política.

Si tenemos en cuenta que el Patrimonio Cultural hace referencia a bienes que pertenecen tanto a la llamada alta cultura como a la cultura popular, esto puede variar su ubicación en el periódico. Siguiendo el esquema propuesto por Rodríguez Pastoriza, la información sobre Patrimonio Cultural, en cuanto a expresión de la alta cultura a través de obras (pintura, escultura, arquitectura) representativas de las 
principales corrientes artísticas a lo largo de la historia, pertenecería a la sección o área de "Cultura". Sin embargo, la información sobre otras manifestaciones de la cultura popular tales como el folclore, las fiestas tradicionales o los objetos artesanos en cuanto a muestras de las formas de hacer en otra época, que también forman parte del Patrimonio Cultural, se incluiría en el área de Sociedad.

Además, según el criterio del origen geográfico por el que una información de cualquier temática que proceda del ámbito geográfico más cercano al de la publicación aparecería publicada en el espacio destinado a local o regional, las informaciones sobre Patrimonio Cultural pueden aparecer también en estas secciones. Por último, el enfoque también puede suponer una variación en su adscripción a una u otra área, dependiendo de si se pone el acento en un aspecto relacionado con la política, podría aparecer tanto en nacional como en local o regional, o bien si se destaca su relación con el turismo, que implicaría su ubicación en el área de Sociedad.

La investigación que se expone a continuación se ha planteado el estudio de la especialización periodística en Patrimonio Cultural por su ubicación en las secciones de "Castilla y León" y "Cultura", qué criterios determinan el reparto de las informaciones en una u otra sección y, en definitiva, si existe o no, aunque sea de forma incipiente una especialización del periodismo cultural centrada en los bienes patrimoniales.

\section{METODOLOGÍA}

El estudio de la ubicación de la información sobre Patrimonio Cultural en las secciones de Castilla y León y Cultura de la prensa regional de esta comunidad autónoma utiliza una metodología que combina el análisis de contenido y la entrevista, lo que permite contrastar los datos obtenidos a través del análisis con la opinión de quienes desarrollan su tarea profesional en los medios analizados.

En cuanto al análisis de contenido, para llevar a cabo este estudio se seleccionaron los dos diarios de mayor difusión de Castilla y León: El Norte de Castilla (Edición Castilla y León) y El Mundo Castilla y León $n^{3}$. El trabajo de campo se desarrolló a lo largo de un año, 2006, lo que permite obtener, así, una representación completa de la información sobre Patrimonio Cultural. En total, se han localizado un total de 1003 unidades periodísticas tras la revisión de 362 ejemplares de ambos periódicos.

Para desarrollar el análisis cuantitativo se aplicó una ficha de análisis a cada una de las 1003 unidades periodísticas localizadas. La ficha, de elaboración propia, incluye 12 variables independientes: Fecha, Sección, Antetítulo, Subtítulo, Data, Tema, Asunto, Fuentes de Información, Género Periodístico, Lenguaje y Rasgos formales. En este trabajo presentamos los resultados del proceso de análisis de contenido de las informaciones sobre Patrimonio Cultural principalmente respecto a las secciones en las que se ubican y también, de cara al estudio de la especialización periodística,

\footnotetext{
${ }^{3}$ Según los datos de la OJD, El Norte de Castilla registró un promedio de tirada de 34.545 ejemplares y de difusión de 29.679 ejemplares. Por su parte, El Mundo de Castilla y León, la edición regional tuvo un promedio de tirada de 20.883 y de difusión de 15.931 en el año 2011. Datos: www.ojd.es [Acceso: $1 / 07 / 2011]$
} 
podemos deducir cuáles han sido los criterios de selección de las informaciones basándonos en datos objetivos obtenidos de otras variables como el número de informaciones sobre un mismo tema o asunto, su distribución a lo largo del año, la sección en que se han publicado, los géneros periodísticos o las fuentes.

La sección del periódico donde se ubica la información tal y cómo la denomina el medio es un dato predeterminado, supone un dato esencial para el estudio de la especialización en el tratamiento del Patrimonio Cultural y puede condicionar el desarrollo de la información, su extensión o tratamiento iconográfico. En este análisis concreto las opciones de secciones incluidas en la ficha de análisis son: "Castilla y León”, “Cultura” y “Opinión”.

El análisis de la ubicación de las unidades periodísticas sobre Patrimonio Cultural publicadas en El Mundo de Castilla y León y El Norte de Castilla tiene en cuenta los dos criterios básicos sobre los que se distribuyen los contenidos en secciones, el criterio geográfico y el temático, además de las diferencias en el diseño y estructura de las secciones de los dos diarios.

El suplemento regional de El Mundo de Castilla y León tiene una división de secciones que distingue únicamente entre "Opinión" y "Castilla y León", pero para el análisis también se tendrá en cuenta la ubicación de las unidades periodísticas en la última que en este periódico sirve de cierre o página cultural. En El Norte de Castilla existe una división más amplia de secciones por lo que se analizan los textos publicados en las tres secciones: "Castilla y León", "Cultura" y "Opinión". También hemos tenido en cuenta si la información tenía presencia en la primera página y, en el caso de $E l$ Mundo, la primera del suplemento de Castilla y León o la primera del diario a nivel nacional. Así mismo se ha registrado si aparece en la primera página del suplemento pero no se desarrolla en el interior, una característica específica de este diario.

El análisis de la ubicación de las unidades periodísticas en las páginas del periódico nos permite observar el grado de especialización en el tratamiento periodístico del Patrimonio Cultural, que será mayor si los textos se ubican en la sección de "Cultura" frente a la más generalista de "Castilla y León" donde los textos se agrupan por su procedencia geográfica y no por criterios temáticos. Asimismo la presencia de textos en las páginas de "Opinión" revela la posición del diario respecto a los diversos tipos de Patrimonio Cultural definidos en el estudio.

Para el análisis de contenido de las unidades relativas al Patrimonio Cultural encontradas en los dos diarios se realizó una división en bloques temáticos atendiendo a dos criterios: el tipo de patrimonio sobre el que versa el texto, según la Ley de Patrimonio Cultural de Castilla y León de 2002, y un segundo criterio, las actuaciones de cara a la conservación y difusión del mismo. En cada uno de ellos hemos destacado además de forma separada el asunto más representativo de este tipo de patrimonio o actuación relacionada con él a lo largo del año de la muestra.

$\mathrm{Al}$ primer criterio, la tipología del Patrimonio Cultural, pertenecen los cinco primeros bloques que agrupan las informaciones sobre Patrimonio Documental (Archivo de Salamanca y Archivos en general), Patrimonio Arqueológico (Atapuerca y resto de 
informaciones denominadas genéricamente en un bloque como Arqueología), Patrimonio Etnológico (Patrimonio Industrial, Fiestas Tradicionales y Canal de Castilla), Patrimonio Lingüístico y Patrimonio de la Iglesia (Las Edades del Hombre). El segundo criterio, las actuaciones para la conservación y difusión del Patrimonio Cultural, sirve para agrupar una serie de informaciones que versan sobre el denominado Patrimonio Histórico-Artístico y sus distintas categorías: bienes muebles (pintura, escultura, orfebrería...) y bienes inmuebles. Se han definido los siguientes bloques: Museos, exposiciones temporales y conmemoraciones culturales, Restauración/Rehabilitación, Denuncia del deterioro de bienes culturales, Difusión y promoción turística del Patrimonio Cultural.

Para completar el análisis de contenido se realizaron cuatro entrevistas con un esquema de entrevista semiestructurada (Vallés, 2009; Anduiza et al, 2009). En El Mundo de Castilla y León se entrevistó a la que fuera redactora de la sección de de Cultura de El Mundo-Diario de Valladolid, Cristina San José de la Rosa, y a la redactora jefe de la sección Castilla y León, María Romero. En El Norte de Castilla se entrevistó a Sonia Andrino, jefa de la sección “Castilla y León” y Angélica Tanarro, jefa de la sección de Cultura.

\section{ANÁLISIS Y DISCUSIÓN}

Los datos resultantes de la aplicación de la ficha de análisis a las 1003 unidades periodísticas muestran diferencias entre los dos periódicos en cuanto a la ubicación de los textos sobre el Patrimonio Cultural según la clasificación elaborada para el estudio pero también algunas concordancias. Respecto al primer bloque temático, la información sobre los bienes patrimoniales según la tipología contemplada en la Ley de Patrimonio Cultural de Castilla y León de 2002, los textos sobre estos tipos de patrimonio se agrupan mayoritariamente en la sección de "Castilla y León", principalmente los referidos a patrimonio documental (Archivo de la Guerra Civil de Salamanca), patrimonio arqueológico (arqueología) y patrimonio lingüístico.

Uno de los criterios por los que la información sobre un tipo de patrimonio aparece en la sección regional es el hecho de que exista una vertiente política que sobrepase a los aspectos culturales del hecho. El caso más claro es el del Archivo de Salamanca, objeto de confrontación entre partidos, gobierno central y regional, pero también tiene este matiz el patrimonio lingüístico o la arqueología, donde se tienen en cuenta aspectos como la inversión pública en obras de conservación de estos bienes o la aparición de restos arqueológicos a raíz de obras de reforma de edificios o mejora de calles.

La sección de "Cultura" de El Norte de Castilla, cuya diferenciación en el periódico es más clara que en El Mundo de Castilla y León, acoge los textos sobre Atapuerca y Las Edades del Hombre. En cuanto al patrimonio etnológico en el decano de la prensa regional se incluye en "Castilla y León" mientras que en El Mundo de Castilla y León aparece con frecuencia en la última dedicada a "Cultura" especialmente las unidades referidas al subapartado de fiestas populares, cuando dispone de apoyo fotográfico, al igual que las unidades sobre Arqueología o sobre Las Edades del Hombre. 
En la sección de "Opinión" se reflejan principalmente los asuntos relacionados con algún tipo de Patrimonio Cultural con una vertiente política como sucede con el Archivo de la Guerra Civil de Salamanca dentro del patrimonio documental y también con el patrimonio lingüístico. En este caso el incremento de textos de opinión en ambos diarios se justifica en los dos primeros meses del año por la confrontación con Cataluña por el Archivo y después con la "percha" de actualidad del 23 de abril, día del Libro y de la Comunidad. Este aumento es especialmente destacado en El Mundo de Castilla y León donde además se publicó un editorial y dos sueltos en los que expone la línea editorial del periódico en la que, al rechazo al traslado de documentos del Archivo de Salamanca a Cataluña, se une la defensa del español.

El patrimonio arqueológico, que es el segundo en cuanto a número de unidades periodísticas, no es objeto de ningún texto en la sección de "Opinión" lo que denota una escasa posición crítica de la prensa regional respecto este tipo de patrimonio. Tampoco existe posición crítica sobre Atapuerca, como demuestra el hecho de que no haya ningún editorial sobre alguno de los asuntos tratados y tan sólo se hayan publicado dos artículos de opinión, uno de ellos firmado por los responsables de la investigación de Atapuerca en la última página de El Mundo, además de otro en las páginas de Cultura de El Norte de Castilla (22/07/06), con el antetítulo "Análisis" firmado por Javier Pérez de Andrés.

La falta de crítica o de adopción de una postura concreta por parte del diario expresada en un editorial se produce incluso pese a que entre las noticias recogidas sobre arqueología se encuentran informaciones que por su carácter de conflicto entre administraciones podrían ser objeto de una toma de posición del medio, como el caso de la rehabilitación del Palacio de los Águila en Ávila como sede secundaria de El Prado en Ávila y la aparición de restos arqueológicos consecuencia de las obras o el rechazo de algunos colectivos a la construcción de un aparcamiento de la plaza de los Bandos en Salamanca por la preocupación por la conservación tanto del patrimonio arqueológico como del conjunto histórico. Tampoco se destinan artículos de opinión a un proyecto inversor de la categoría del complejo de la Evolución Humana que se inicia con el Museo de la Evolución Humana con una inversión global de entorno a los 190 millones de euros.

Las Edades del Hombre, una muestra consolidada en el tiempo, es también objeto de artículos en la sección de "Opinión" firmados en todos los casos por periodistas colaboradores habituales de los diarios que se centraron en la vertiente cultural o turística de la muestra. Dado que la Iglesia organiza estas exposiciones con una función catequética o evangelizadora, además de la conservación y difusión de su patrimonio, hubiera sido lógico esperar la presencia en estas páginas de textos de algún teólogo o especialista en arte religioso que expusieran argumentos, vivencias o impresiones, o bien algún artículo del comisario de la muestra o el obispo presidente de la Fundación de Las Edades del Hombre. Sin embargo no encontramos ninguna firma relacionad directamente con la muestra y ninguno de los dos diarios expresó su postura respecto a la exposición a través de editoriales o sueltos. Por lo tanto, la visión de la exposición de Las Edades del Hombre que exponen los articulistas se centra en 
Patrimonio Cultural - Periodismo especializado - Prensa - Cultura - Tratamiento de la información

su vertiente cultural o turística más que en el significado religioso que pretende la organización.

La ubicación en las páginas de los dos diarios analizados de las unidades periodísticas del segundo bloque temático, las referidas a la conservación y difusión del Patrimonio Cultural ofrece datos que apuntan a una mayor especialización en el tratamiento periodístico de estos contenidos. En general, las páginas de la sección de "Cultura" en El Norte de Castilla y su equivalente en el suplemento regional de El Mundo, la última página, acogen las unidades periodísticas referidas a actividades de difusión y conservación del patrimonio con una vertiente artística o de divulgación del conocimiento. Por su parte la sección de "Castilla y León" en el decano de la prensa regional y las páginas interiores de El Mundo de Castilla y León reúnen informaciones relacionadas con aspectos económicos, organizativos o estadísticos de las actividades de difusión y conservación del Patrimonio Cultural.

El principal ejemplo de esta separación de contenidos lo encontramos en el bloque de difusión y promoción turística. En los dos diarios el factor economicista del turismo aleja estos asuntos de las páginas de la sección de "Cultura", o de la última página de El Mundo de Castilla y León, y se incluyen mayoritariamente en la sección de "Castilla y León" en El Norte de Castilla o en páginas interiores del suplemento en El Mundo. Por otra parte, los asuntos más relacionados con la difusión, tales como las publicaciones, o cuyas fuentes son fundaciones culturales suelen publicarse en páginas de "Cultura", especialmente en El Norte de Castilla.

También en el bloque relativo a museos, exposiciones temporales y conmemoraciones culturales encontramos muestras de esta división de contenidos. Las unidades periodísticas sobre exposiciones e publican mayoritariamente en ambos diarios en el espacio destinado a "Cultura" poniendo el énfasis en su carácter artístico. La información sobre el V Centenario de la muerte de Colón se divide casi al $50 \%$ en los dos periódicos entre la sección de "Castilla y León", donde se recogen unidades periodísticas relativas a cuestiones organizativas y estadísticas de visitantes, y la de "Cultura" donde se informa del contenido de las exposiciones y los bienes culturales que las integran. La información sobre museos, sin embargo, tiene una división menos clara. En El Mundo de Castilla y León aparece principalmente en el interior del suplemento, el equivalente a la sección de "Castilla y León". En El Norte de Castilla, aunque se publicaron más unidades periodísticas en "Cultura", aparecen en "Castilla y León" aquellas referidas a centros museísticos de menor condición artística como el Museo del Comercio de Salamanca o aquellos situados en provincias en las que el periódico carece de edición propia y de cuya redacción se encargan los colaboradores que habitualmente trabajan para la sección de "Castilla y León".

La sección de "Castilla y León" acoge la mayoría de los textos sobre denuncia del deterioro de bienes culturales y restauración y rehabilitación en ambos diarios, probablemente porque se destaca en estas actuaciones su aspecto socioeconómico por encima del cultural. En El Mundo de Castilla y León también aparecen estas informaciones en la última página de temas culturales, en este caso podría señalarse que la inclusión de fotografías favorece este emplazamiento. 
Los datos extraídos del análisis de contenido coinciden en gran parte con las respuestas de las entrevistas realizadas a profesionales de ambos diarios que denotan una mayor especialización en El Norte de Castilla que El Mundo de Castilla y León. Una de las razones de esta diferencia es la situación de recorte, tanto de páginas como de plantillas, de la edición regional de El Mundo lo que redunda en una menor especialización de los redactores y una apuesta por el periodista generalista, capaz de cubrir muy diferentes temas. En El Mundo de Castilla y León en 2011 la sección de "Castilla y León" tenía 5 periodistas, ninguno especializado en Cultura, y corresponsales en provincias. Por su parte, El Norte de Castilla dispone de una plantilla mucho más amplia: 6 redactores en "Cultura" además de colaboradores especializados y en la sección de "Castilla y León" trabajan tres redactores y colaboradores de cada una de las nueve provincias.

También denota la menor especialización en El Mundo de Castilla y León las respuestas de las entrevistadas en este diario sobre los criterios de ubicación de las informaciones sobre Patrimonio Cultural en el suplemento regional donde la última página se dedica a asuntos culturales. La redactora jefe de "Castilla y León", María Romero, señala que ambas secciones no son incompatibles en algunos temas, lo que apunta a una gran permeabilidad de los asuntos relativos al Patrimonio Cultural. En este sentido, la redactora de El Mundo-Diario de Valladolid, Cristina San José, apunta a cierta ambigüedad en los matices de una información para su publicación en la sección de Castilla y León y señala que también depende de la agenda del día.

De las palabras de Sonia Andrino, jefa de la sección "Castilla y León" y de Angélica Tanarro, jefa de "Cultura" de El Norte de Castilla se deduce que el elemento esencial que distingue una de otra es la política, si el asunto relacionado con el Patrimonio Cultural tiene una vertiente política, ya sea de enfrentamiento entre partidos o de obras de restauración, se ubicará en la sección de "Castilla y León". Por el contrario, si el asunto a tratar tiene una dimensión cultural, como un congreso de expertos, una exposición o la presentación de un libro, aparecerá en las páginas de "Cultura", lo que muestra un cierto grado de especialización en Patrimonio Cultural en el decano de la prensa regional.

La crisis económica es mencionada las entrevistadas cuando se les pregunta por el futuro de la especialización periodística junto a otro factor que incide en el futuro del periodismo, las nuevas tecnologías y aquí encontramos diferencias de criterio entre las entrevistadas. La redactora jefe de Castilla y León de El Mundo, María Romero, apunta a internet como un factor que incidirá en la necesidad de una mayor especialización mientras que la jefa de Castilla y León de El Norte de Castilla, Sonia Andrino, contrapone la figura del periodista especializado con el periodista multimedia.

La situación económica es el factor que marcará el futuro de la especialización en la profesión para la redactora de cultura de El Mundo-Diario de Valladolid, Cristina San José, que coincide con la jefa de Cultura de El Norte de Castilla, Angélica Tanarro, que considera que la especialización sería necesaria pero que en la situación actual será cada vez menor. 


\section{CONCLUSIONES}

La amplitud del concepto de Patrimonio Cultural dificulta, como hemos visto, su adscripción a un área y más concretamente al área de Cultura convirtiéndose en una materia transversal que puede cambiar de ubicación según el enfoque dado a la información aunque la relevancia de los bienes incluidos en el Patrimonio Cultural y la especificidad de la materia requerirían un trato especializado.

El análisis de las 1.003 unidades periodísticas sobre Patrimonio Cultural publicadas en El Mundo de Castilla y León y El Norte de Castilla en 2006 no se han podido establecer criterios homogéneos por los que las informaciones sobre un tipo de patrimonio o una actividad relacionada con un bien cultural se publiquen en la sección "Castilla y León" y "Cultura", aunque el análisis de contenido y las entrevistas permiten deducir los criterios de reparto de estas informaciones en una u otra sección, criterios que son variables según el periódico y según los matices de la información.

El elemento esencial que sirve para delimitar la ubicación en una u otra sección de un asunto relacionado con el Patrimonio Cultural es la política. Si el hecho noticioso relativo al patrimonio tiene una vertiente política, se ubicará en la sección de "Castilla y León". Este matiz político puede proceder de que un enfrentamiento entre gobierno y oposición por algún asunto cultural, caso del archivo de Salamanca o las obras de restauración del Palacio de los Águila en Ávila. Hay que tener en cuenta que en el año de toma de datos, 2006, el gobierno nacional y el regional estaban regidos por partidos distintos, el PSOE en el gobierno central y el PP en Castilla y León.

Otra vertiente que determina, por regla general, la ubicación en la sección Castilla y León de las unidades periodísticas sobre Patrimonio Cultural es la económica, es decir, las repercusiones que pueda tener en la economía regional la conservación y difusión de los bienes culturales. En la sección de Castilla y León se publican unidades periodísticas en las que se destaca, por ejemplo, el presupuesto de inversión en una obra de restauración de un monumento por parte de la administración pública (sea esta regional, principalmente, nacional o provincial) o las repercusiones económicas de la celebración de actos culturales con motivo de una conmemoración histórica o de una exposición temporal como Las Edades del Hombre. Según el criterio de las repercusiones económicas de las actuaciones relativas al Patrimonio Cultural, generalmente las unidades referidas a la promoción del turismo cultural en la región se publican en la sección de Castilla y León que agrupa los textos atendiendo a un criterio geográfico.

Por el contrario, si el asunto a tratar tiene una dimensión cultural, como un congreso de expertos, una exposición de la que se detallan las piezas mostradas o la presentación de un libro, aparecerá en las páginas de "Cultura". Los matices en cuanto a la distribución de los contenidos en secciones coinciden con los criterios que señala Rodríguez Pastoriza (2006) por los que las realizaciones de la alta cultura, las bellas artes en cuanto al Patrimonio Cultural se refiere, se incluyen en la sección de Cultura, mientras que otras manifestaciones relacionadas con la cultura popular, el turismo y la 
gastronomía aparecen la sección de Sociedad, en el caso de los diarios analizados, la sección de Castilla y León.

La ordenación de los contenidos en secciones también depende, por cuestiones relativas a la organización interna de cada periódico, de la agenda del día y del propio diseño del diario. En el caso de El Norte de Castilla, el hecho de que exista una sección diferenciada de Cultura y dotada de una amplia plantilla de redactores (6 periodistas además de colaboradores especializados en música, literatura, etc.) facilita la ubicación de las unidades periodísticas sobre Patrimonio Cultural en esta sección. El diseño de El Mundo de Castilla y León, donde no se diferencia una sección de Cultura, sumado al hecho de que no exista ningún redactor dedicado en exclusiva a temas culturales en la plantilla de la edición regional compuesta por cinco redactores, hace que exista menor grado de especialización.

El Patrimonio Cultural recibe en ambos diarios tratamiento a través de géneros de "Opinión", en las páginas de esta sección. La opinión del periódico, manifestada a través de editoriales y sueltos, se expresa, casi exclusivamente en los dos periódicos analizados, en relación al traslado de documentación del Archivo de la Guerra Civil de Salamanca a Cataluña, cuestión que fue ampliamente tratada en las páginas de los dos diarios regionales.

Cabe concluir, por tanto, del análisis de contenido y las entrevistas realizadas que la especialización periodística en Patrimonio Cultural en El Norte de Castilla y El Mundo de Castilla y León no está delimitada ni en la organización de la plantilla ni en la ordenación de los contenidos en las secciones. Pese a ello, el grado de especialización es mayor en El Norte de Castilla que en El Mundo de Castilla y León. El decano de la prensa regional tiene una sección de "Cultura" con su propia plantilla que se reparte los temas de la sección incluyendo el Patrimonio Cultural, asuntos de los que se encarga habitualmente un redactor.

Respecto a la ordenación de contenidos en secciones, el tratamiento periodístico del Patrimonio Cultural no es especializado en cuanto a su ubicación en una sección fija. Del análisis de contenido y de las entrevistas realizadas no se ha podido extraer un criterio unánime sobre su publicación en la sección de Cultura o en la sección de Castilla y León aunque se ha detectado que su inclusión en una u otra sección depende de los matices de la información. Si se trata de un acontecimiento con una vertiente política o económica el criterio determina que aparezca en la sección Castilla y León. Si está relacionado directamente con las Bellas Artes o con la publicación de libros, ocupará el espacio en la sección de Cultura.

La especialización en Patrimonio Cultural, con los datos de esta investigación, es incipiente y escasa. Si tenemos en cuenta el relevante papel que desempeñan los medios de comunicación en la difusión cultural que puede contribuir a la concienciación de la sociedad del valor intrínseco de los bienes culturales como elementos definitorios de la identidad colectiva, de la cultura y la historia de un territorio, sería deseable que los contenidos en prensa relacionados con el Patrimonio 
Patrimonio Cultural - Periodismo especializado - Prensa - Cultura - Tratamiento de la información

Cultural ocuparan un espacio fijo y predeterminado en la sección especializada de Cultura. Este espacio definido en la prensa permitiría primar los aspectos históricos y artísticos de los bienes culturales, su valor intrínseco, sobre consideraciones políticas, partidistas y económicas que desvirtúen el valor de este legado cultural.

\section{BIBLIOGRAFÍA}

Anduiza, E.; Crespo Martínez, I. y Méndez Lago, M. (2009). En Metodología de la Ciencia Política (p. 87 y ss). Cuadernos Metodológicos, 28. Madrid: Centro de Investigaciones Sociológicas.

BOE (1985): Ley de Patrimonio Histórico Español. Ley 16/1985, de 25 de junio, de Patrimonio Histórico Español. B.O.E. $\mathrm{n}^{\mathrm{o}} 155$ de 29 de junio de 1985. En: http://www.boe.es/

Casals Carro, M. J. (2005). Periodismo y sentido de la realidad. Teoría y análisis de la narrativa periodística. Madrid: Fragua.

Fernández de Paz, E. (2006). De tesoro ilustrado a recurso turístico: el cambiante significado del patrimonio cultural. En Pasos. Revista de Turismo y Patrimonio Cultural, vol. 4, p. 1-12.

Hernández, F. (2002). El patrimonio cultural: la memoria recuperada. Gijón: Trea

Herrero Aguado, C. (2002). Las secciones como principios de ordenación temática de los contenidos en el periodismo especializado. En Prensa y Periodismo especializado (historia y realidad actual). (pp. 25-40) Guadalajara: Actas del Congreso de Prensa y Periodismo Especializado celebrado el 8-10 de mayo.

Junta de Castilla y León (2002). Ley de Patrimonio Cultural de Castilla y León. Ley 12/2002, de 11 de junio, BOCYL no 139 de 19 de julio de 2002 y BOE no 183 de 1 de agosto de 2002. Disponible en: http:/ / bocyl.jcyl.es/

Llano, R. (2008). La especialización periodística. Madrid: Tecnos.

Leyton Rivas, R. (2007). El Patrimonio Cultural en la Formación del Periodista. En. REpresentaciones. Periodismo, Comunicación y Sociedad. Universidad de Santiago, núm. 2, p. 107-114.

Mateos Rusillo, S. M. (coord.) (2008). La comunicación global del patrimonio cultural, Gijón: Trea

Meneses Fernánez, M. D. (2011). Periodismo, medias y patrimonio: de la curiosidad arqueológica y paleontológica a la ciencia y al desarrollo zonal. En: Estudios sobre el mensaje periodístico, vol. 17, núm. 2, p. 365-381.

Mercado Sáez, M. T. (2006). Informar sobre Patrimonio Cultural: una especialización necesaria. En Prensa y Periodismo Especializado 3, Vol. 2 (pp. 223-233). Guadalajara: Editores del Henares. 
Rodríguez Martínez, R. (2010). Explicar y difundir la cultura. En I. Camacho Markina (coord.), La especialización en periodismo. Formarse para informar (pp. 162-177) Sevilla: Editorial Comunicación Social.

Rodríguez Pastoriza, F. (2006). Periodismo Cultural. Madrid: Editorial Síntesis.

Ruiz Zapatero, G. y Mansilla Castaño, A. M. (1999), “L'Arqueologia en els mitjans de comunicació. Materials per una reflexió crítica sobre la divulgació del pasta". En: COTA Zero, núm. 15, p. 42-62

Rodríguez Temiño, I. (2007). Arqueología a diario. En PH Cuadernos 21. Patrimonio Cultural y Medios de Comunicación. Sevilla: Instituto Andaluz de Patrimonio Histórico, Consejería de Cultura de la Junta de Andalucía.

Tugores, F. y Planas, R. (2006). Introducción al patrimonio cultural. Gijón: Trea.

UNESCO (2003): Convención para la salvaguardia del patrimonio cultural inmaterial de París. En: http://unesdoc.unesco.org/images/0013/001325/132540s.pdf [Consulta: $1 / 06 / 2012]$

UNESCO (1972): Convención sobre la protección del Patrimonio mundial, cultural y natural de la Humanidad. En: http://whc.unesco.org/archive/convention-es.pdf [Consulta: 01/07/2012]

Valles, M. S. (2009). Entrevistas Cualitativas. Cuadernos Metodológicos, 32. Madrid: Centro de Investigaciones Sociológicas.

VVAA (2007). PH Cuadernos 21. Patrimonio Cultural y Medios de Comunicación. Sevilla: Instituto Andaluz de Patrimonio Histórico, Consejería de Cultura de la Junta de Andalucía. 


\section{María Monjas Eleta}

Doctora por la Universidad de Valladolid (2012), Licenciada en Ciencias de la Información, Periodismo, por la Universidad Pontificia de Salamanca y Máster de Radio de la Universidad Complutense de Madrid. Profesora asociada en la Universidad de Valladolid. Investigadora a tiempo completo del proyecto regional “ $\mathrm{La}$ imagen de Castilla y León en la prensa de referencia" y del proyecto nacional "El infoentretenimiento político en televisión e internet". Miembro del equipo del Proyecto de Innovación Docente en TIC y multidisciplinareidad en el Grado en Periodismo: medios culturales especializados. 